\title{
HEDGE ALGEBRAS, THE SEMANTICS OF VAGUE LINGUISTIC INFORMATION AND APPLICATION PROSPECTIVE
}

\author{
Cat Ho Nguyen ${ }^{1, ~ *}$, Thai Son Tran ${ }^{1}$, Nhu Lan Vu ${ }^{1,2}$ \\ ${ }^{1}$ Institute of Information Technology, VAST, 18 Hoang Q Viet, Cau Giay, Hanoi \\ ${ }^{2}$ Thang Long University, Nghiem Xuan Yem, Hoang Mai, Ha Noi \\ *Email: ncatho@gmail.com
}

Received: 18 November 2014; Accepted for publication: 4 June 2015

\begin{abstract}
The report aims to show that hedge algebras model actually the proper qualitative semantics of words of linguistic variables based on the argument that the inherent qualitative semantics of words should be expressed through the order relationships between the words in their respective variable domains induced by the word semantics, as it is required by decision making of human daily lives. This makes the hedge algebra based approach to the word semantics quite different to the existing approaches and become the only approach that can immediately deal with the natural qualitative semantics of words. We explain clearly and systematically distinguished features and properties of this approach to show that these seem to make the approach to be sound and to ensure its effectiveness in initial applications under consideration. This approach seems to be promising for development of hedge algebra-based method to solve problems in various application fields. For illustration, we will give a short overview of effective results of the initial applications of hedge algebras in the fields of knowledge based systems and of fuzzy control.
\end{abstract}

Keywords: order based semantics; fuzziness of word; fuzzy set based semantics, fuzzy rule based system; classification; fuzzy control.

\section{INTRODUCTION}

Uncertainty information, including fuzzy linguistic information, appears in almost areas of human society and of technology and, therefore, any theories of uncertainty can find a wide range of applications in many distinct fields. The fuzzy set theory is one of such theories, whose development is motivated by the semantics of words and human capabilities in handling such information, as stated by Zadeh [1]: "Humans have many remarkable capabilities. Among them there are two that stand out in importance. First, the capability to converse, communicate, reason and make rational decisions in an environment of imprecision, uncertainty, incompleteness of information and partiality of truth. And second, the capability to perform a wide variety of physical and mental tasks without any measurements and any computations." The fuzzy set 
theory provides a mathematical formalism to deal with the word semantics simulate such human capabilities in problem solving.

However, to exhibit the specific role of hedge algebras and their prospective applications, we should start our study with making the semantics of words explicitly and formally defined. In the fuzzy set theory, words are represented as fuzzy sets, which are a generalization of crisp sets. Note that the inherent semantics of words has still not been formally defined in the framework of fuzzy sets, meanwhile one usually interprets the considered fuzzy sets as representing the semantics of the respective words assigned to them without any explicit basis to justify why these words but not the other ones can more relevantly be assigned to them. In contrast, the theory of hedge algebras is developed by starting with a formalized definition what is the own semantics of words and their fuzziness, an essential characteristic of any fuzzy data. In this overview, we argue more explicitly that "Normally, the meaning of a word or a phrase is a collection of objects or phenomena present in the real world that the terms or phrases point at" and, naturally, "The presence of these terms (vague words) in natural language aims to compare properties of distinct items in terms of words. This semantics seems to be very crucial for human decision making as it will be discussed next. ..." [2]. That is, we will argue that the semantics of (vague) words must point at order relationships between the words of a linguistic variable (attribute). This seems to be very natural and essential and it makes the algebraic approach essentially different from the fuzzy set based approach, which sometime we call "the analytic approach", in modeling the word semantics and in simulating human capabilities mentioned above. We will demonstrate in an obvious manner that the theory of hedge algebras forms a sound mathematical and logical foundation to manipulate immediately words in problem solving. It is natural to expect that the sounder basis of an approach, the more advantageous and effective it may offer. At the same time, we will exhibit initial applications of distinct fields, e.g. classification, decision making and fuzzy control using fuzzy rule bases systems (FRBSs), which contribute to show the soundness and the effectiveness of the algebraic approach.

The rest paper is organized as follows. In Section 2 we present why hedge algebras form a sound approach to the semantics of words. Section 3 devoted to explain why this approach can bring out the effectiveness in the applications under consideration and to expose shortly their results. Section 4 offers for main conclusions.

\section{HEDGE ALGEBRAS - A SOUND MATHEMATICAL STRUCTURE FOR MODELING AND HANDLING THE PROPER WORD SEMANTICS}

\subsection{What is the semantics of vague words?}

To show hedge algebras (HAs) to be a sound mathematical structure to deal with to the semantics of words, we start with discussion about the semantics of words since it is crucial and elementary concept motivating the introduction of fuzzy sets and, then, HAs.

Semantics of formal expressions (syntax) is an important abstract concept of formal logics. Words in a natural language viewed as as symbolic strings conveying their meaning, which point at definite things, facts or phenomena in reality, are used to communicate between people in a community or to do reasoning. For example, the meaning of the symbolic string "river" in English is a collections of items in the real world that the community in this reality together point out that they are "river". Similarly, we can explain the meaning of "tree", "green", "rose flower", and so on. Although the semantics of such words is sophisticated, it may still be very easy for us to explain what the meaning of such words is, as they point at concrete items in the 
reality. It seems to be much more difficult to define the semantics of vague terms such as "true", "young", "beautiful", ..., since they do not still indicate concrete items in the real world. So, what things in the reality they point at?

To define the semantics of this kind of words, first, the authors of the study do not think that they point at "fuzzy sets", because no any individuals of a human community think of a collection of items in reality that the fuzzy sets describe when reading or saying these words. To argue what is the meaning of, for example, the symbolic expression "young" in English, we try to imagine what the word "young" points at. Assume that "young" is a words of the attribute AGE (a linguistic variable). Even then we can observe that it still does not point at concrete items of the real world, because he does not know "young" points at people, animals or other things. To discuss about this it is useful for us to imagine in which way a language has been taken shape along with the existence and development of a human community. We can see that language serves for cognition of reality and, especially, for the decision making of human beings. We recognize that life of a human being, and even of an animal, comprises a consecutive series of decisions. The nature of a decision is to choose a more preferable alternative among several ones. That is comparability between properties of items is essential and crucial for decision making. As a consequence, in natural languages, as vehicles convey semantics for communicating and doing reasoning of human beings, should have elements (words, phrases, linguistic hedges, ...) to describe preferable alternatives based on comparison between their properties with respect to certain decision criteria. Thus, it implies that words in a context of a linguistic variable (attribute) used to describe properties of items in the real world are comparable, i.e. the semantics of words of a variable, generate an order relation on the worddomain of the variable. So, we can recognize that words in this context point at their order relationships with the other ones in the same context, noting that two words may even be incomparable.

This viewpoint of the word semantics seems to be very essential and fundamental. Once term-domains have their own structure, modelling the word semantics, one should start with this structure to develop a formalism. After all, the existence and development of a human being or a human community, or even of an animal, in a real world is just a real and objective environment in which language accompanying with a community has taken form and, as a consequence, involves elements used to describe preferable features of alternatives in the reality serving for decision making.

\subsection{Hedge algebras - A sound mathematical semantic structure of term-domains of variables}

Restricting ourselves to the context of a linguistic variable, based on this viewpoint of the word semantics, every word-domain can be viewed as an order-based structure in which the meaning of a word is described by a collection of its order relationships with the other words of the word-domain. Thus, any mathematical theory developed to model the semantics of words of a variable should be able to deal with this semantics, e.g. should preserve the order-based structure of the word-domain. It can easily be seen that the fuzzy set theory does not preserve these order-based structures.

Hedge algebras were introduced and developed to model and represent this semantics of words by considering word-domains of linguistic variables as algebras and by trying to discover the semantic properties of words in terms of the "inherent" semantic order relation of the worddomains $[3-6,7,8]$. Because term-domains in natural languages have their own inherent order- 
based structure, we may expect that many interesting semantic properties of words can be discovered and, it can be observed that, they cannot be formulated in the framework of the fuzzy set theory.

Formally, every word-domain of a variable $X, \operatorname{Dom}(X)$, can be considered as an abstract algebra, $\mathcal{A} X=\left(X, G, C, H_{I}, \leq\right)$, where $X$ is a word-set of $X ; \leq$ is an order relation on $X ; G$ is a set of two generators, one is called the negative primary term $c^{-}$and the other the positive primary term $c^{+}$satisfying $c^{-} \leq c^{+}$, for instance, small $\leq$big; $C=\{\boldsymbol{0}, W, \boldsymbol{1}\}$ is the set of constants, in which 0 and 1 are, respectively, the least and the greatest term in $(X, \leq)$ and $W$ is the neutral term, e.g. "medium", "middle-age", .., satisfying $\boldsymbol{0} \leq c^{-} \leq W \leq c^{+} \leq \boldsymbol{I} ; H_{I}=H \cup\{I\}$, where $H$ is a set of hedges of $X$, regarded as unary operations, and $I$ is the identity of $X$. So, every term $x$ in $X$ can be represented as a string expression, i.e. either $x=c$ or $x=h_{m} \ldots h_{1} c$, for some $c \in G \cup C$ and $h_{j} \in H, j=1, \ldots, m$, and it is called (string) representation of $x$, which is quite similar to words expressions in natural (English) languages, e.g. "very rather true".

In this formalized structure, many natural properties can be formulated and discovered [3], for instance, the following:

First, it is compatible with the comparability demand for human decision making, the action effect of every hedge $h$, e.g. $h$ is "very", "little", or "extremely" ..., when acting on a word $x$, causes order relationships between the resulting word $h x$ and the operand word $x$, i.e. we have either $x \leq h x$, or $x \geq h x$. If $h x=x$, then $x$ is a fixed point and, then, finite hedge algebras can be assumed.

It is interesting that in terms of $\leq$, we can define the following concepts, but not the only ones, and they cannot be discovered in the fuzzy set theory framework (refer to [2, 3, 5, 9]):

-Algebraic sign of the primary words $c^{-}$and $c^{+}, c^{-} \leq c^{+}$, representing their inverse semantic tendencies: $\operatorname{sign}\left(c^{-}\right)=-1$ and $\operatorname{sign}\left(c^{+}\right)=+1$. For example, fast and slow have inverse tendencies and $\operatorname{sign}($ slow $)=-1$ and $\operatorname{sign}($ fast $)=+1$.

-Algebraic sign of the hedges: firstly, as discussed above, every hedge either increases or decreases the order based semantics of an atomic (primary) word and, hence, it has an "algebraic" sign. So, the set $H$ of hedges of interest is partitioned into the set of positive hedges, $H^{+}=\left\{h \in H: h c^{+}>c^{+}\right\}$, and the set of negative hedges, $H^{-}=\left\{h \in H: h c^{+}<c^{+}\right\}$. Secondly, every hedge has its semantic effects with respect to any other ones. For instance, $\operatorname{sign}(L, V)=-1$, where $L$ and $V$ stand for Little and Very, respectively, as $L$ decreases the effect of $V$, e.g. true $\leq L_{-} V_{-}$true $\leq V_{-}$true, whereas $\operatorname{sign}(V, L)=+1$.

-Hedge inheritance, which describes the own function of the hedges that they only modify or intensify the semantics of a given word, while inheriting a specific key semantics of the word they act on. In terms of $\leq$, it can be formulated as follows (next, $R$ stands for Rather):

(i) $h \neq k \& h x \leq k x \Rightarrow h^{\prime} h x \leq k^{\prime} k x$, for all $h, k \in H_{I}$. For instance, $L \_t r u e \leq R \_t r u e \Rightarrow$ $h L \_$true $\leq k R \_$true; and

(ii) $v \notin H_{I}(u)$ and $v \leq u(v \geq u) \Rightarrow v \leq h u(v \geq h u)$, for every $h \in H_{I}$.

- Word independency: If $u, v$ are independent, i.e. $u \notin H_{I}(v)$ and $v \notin H_{I}(u)$, then $x \in H_{I}(u) \Rightarrow$ $x \notin H_{I}(v)$.

The above properties that originate only from the natural semantics of words are basis to establish an axiom system for word-domain structures, including in particular linear ones, and, then, we obtain algebraic structures, called Hedge Algebras (HAs). Note that, we restrict 
ourselves to the linear structures of word-domains in this study. So, hedge algebras provide a strict mathematical formalism to handle immediately words as linguistic values of variables and it is interesting that it is sufficient to develop hedge algebras to become a rich enough theory to deal with linguistic fuzzy information for problem solving in an uncertainty environment.

\subsection{Quantification of hedge algebras and the quantitative semantics of words}

Fuzzy sets assigned by human user to words can be interpreted as being quantitative semantics of words and one argues that they represent the fuzziness of words. Then, the fuzziness of words was defined pointwise based on the membership values of their fuzzy sets: every value taken in $[0,1]$ represents an uncertainty degree of the membership of an element of the variable universe of discuss that attains the unit 1 for the element membership degree value equal to $1 / 2$, the value 0 for the element membership degree values equal to 0 or 1 and a value in between 0 and 1 for the remaining element membership degree values. Then, the fuzziness measure of a word is measured by the sum of the uncertainty degrees of the membership values of its assigned fuzzy set. That is, instead dealing with the fuzziness with words, one had to deal with the fuzziness of their assigned fuzzy sets.

It can be seen that there is no formal linkage of the word semantics with the fuzzy sets assigned by the user to the words of interest, whereas the fuzziness of words is defined by their fuzzy sets, i.e. it depends on the fuzzy set assignment by the user, but not on the words themselves. Therefore, there is an obvious gap between the actual semantics of words and their fuzzy set based semantics assigned to them by the user. This may causes some problems, e.g. the definition of the fuzziness of words based on the membership values of their fuzzy sets might not be represent the proper fuzziness of words. Similarly, once the fuzzy set based semantics of words cannot be formally defined based on their own inherent qualitative semantics, there is no basis to ensure that the fuzzy sets can properly represent the word semantics, while the fuzzy set theory aims to simulate the remarkable capabilities of human beings in processing and reasoning with linguistic information.

Up to now, HAs can be regarded as the only theory that manipulates directly on words and their qualitative semantics and they form an algebraic approach to the word semantics, versus the fuzzy set theory which is regarded as an analytic approach developed utilizing the structure of the analytic function space. In HA approach, the order-based qualitative semantics of words determines formally and algorithmically their quantitative semantics. This is very important, because it forms a formal basis to develop methodologies to deal simultaneously with the qualitative semantics of words and their quantitative one, including the fuzzy set based semantics of words, and this can be done only when word-domains are formalized. This is reasonable and compatible with the nature of the word semantics: words of natural languages convey their meaning that are mapped to their respective items in the reality, but not conversely.

This formal basis comprises the following quantification characteristics:

\subsubsection{Fuzziness model, fuzziness measure and fuzziness intervals of words}

In the algebraic approach, fuzziness of words plays a centric role in defining not only the distinct characteristics of words presented in this section, but also the fuzzy set based semantics of words. This seems to be compatible with the fuzziness nature of the fuzzy linguistic information and this contributes to make this approach quite different from the analytic one. 
Consider a linear HA $\mathcal{A} X=\left(X, G, C, H_{I}, \leq\right)$ of a linguistic variable $X$. To define the fuzziness of a word, we try to explain why the word "green" is fuzzy. One may envision that words become inexact or fuzzy because every natural language comprises only a finite number of words, while they are used to describe infinite items of the real world. In practice, "green" points at maybe infinite instances of colours that a human community call "green", as they still contain a specific key feature of "green". Following this observation, we now consider a vague word $x$ of $X$. By the hedge inheritance, $H_{I}(x)$ consists of all terms that still inherit a specific genetic key semantics of $x$. Similarly as the fuzziness of "green", $H_{I}(x)$ can be interpreted as to be the fuzziness model of $x$ ([7,8]. The set $\left\{H_{I}(x): x \in X\right\}$ forms a specific structure that can be viewed as a neighbourhood basis of a topology in a general sense and as a basis to define the concept of fuzziness measure.

To introduce an axiom system of fuzziness measure $f m$ of $X$, we assume $\mathcal{A} X$ to be free, i.e. $h x \neq x$, for $\forall x \in X$ and $\forall h \in H$, and let us consider an order-isomorphism $f: X \rightarrow[0,1]$, i.e. it is an one-to-one mapping and preserves the order relationships of $X$. In addition, it is necessary to assume that the image $f(X)$ of $X$ is dense in [0,1]. Our idea is that the fuzziness measure $f m$ is defined for every $x$ as to be $f m(x)=\left|\operatorname{closure}\left(f\left(H_{I}(x)\right)\right)\right|$, the diameter of closure $\left(f\left(H_{I}(x)\right)\right)$. This and the structure of $\left\{H_{I}(x): x \in X\right\}$ suggest us to introduce the following axioms of $f m$ :

$(f m l) f m(c)+f m\left(c^{+}\right)=1$ and $\sum_{h \in H} f m(h u)=f m(u)$, for $\forall u \in X$.

( $f m 2) \forall x, y \in X, \forall h \in H, \frac{f m(h x)}{f m(x)}=\frac{f m(h y)}{f m(y)}=\mu(h)$, called the fuzziness measure of $h$ (hedge).

(fm3) Putting $\alpha=\Sigma\left\{\mu(h): h \in H^{-}\right\}$and $\beta=\Sigma\left\{\mu(h): h \in H^{+}\right\}$, we have $\alpha+\beta=1$.

The set closure $\left(f\left(H_{I}(x)\right)\right)$ defines an interval $\mathfrak{I}(x) \subseteq[0,1]$, whose values can be considered as compatible with the semantics of $x$ and, hence, it is called the fuzziness interval of $x$. Evidently, we have $|\mathfrak{I}(x)|=f m(x)$ and, since $f$ is isomorphic, we have $h x \leq k x \& h x \neq k x \Rightarrow \mathfrak{I}(h x)$ $\leq \mathfrak{I}(k x), x \in X$.

So, the concepts examined in this section seem to be very natural and they have close relationships with each other. In addition, it can be seen from $(f m l)$ and $(f m 2)$ that $f m$ and the fuzziness intervals are completely determined by giving the values of $f m(c)$ and $\mu(h), h \in H$, called the fuzziness parameters of $X$. They are the parameters for tuning to find optimized vague words.

\subsubsection{Semantically quantifying mappings of $\mathcal{A} X[6]$}

We have presented above that a given isomorphism $f$ produces a fuzziness measure $f m$ and the fuzziness intervals of words, $\mathfrak{I}(x), x \in X$. In turn, a given $f m$ can induce an isomorphism, denoted by $v_{f m}$, called Semantically Quantifying Mapping (SQM). The values of an SQM are called numeric semantics of the respective words. $v_{f m}$ is defined recursively as follows:

$(\mathrm{SQM} 1) v_{f m}(W)=\theta=f m(c), v_{f m}\left(c^{\top}\right)=\theta-\alpha f m\left(c^{\top}\right)=\beta f m\left(c^{\top}\right), v_{f m}\left(c^{+}\right)=\theta+\alpha f m\left(c^{+}\right)$.

(SQM2) $v_{f m}\left(h_{j} x\right)=v_{f m}(x)+\operatorname{Sgn}\left(h_{j} x\right)\left\{\left[\sum_{i=\operatorname{sgn}(j)}^{j} f m\left(h_{i} x\right)\right]-\omega\left(h_{j} x\right) f m\left(h_{j} x\right)\right\}$

where $\omega\left(h_{j} x\right)=\frac{1}{2}\left[1+\operatorname{Sgn}\left(h_{j} x\right) \operatorname{Sgn}\left(h_{p} h_{j} x\right)(\beta-\alpha)\right] \in\{\alpha, \beta\}$, for all $j \in\left[-q^{\wedge} p\right]=\{j:-q \leq j \leq p \& j \neq 0$ \} .

Thus, SQMs can again be determined when providing the fuzziness parameter values of $X$. 


\subsection{Semantics core of words and enlarged hedge algebras [10]}

In fuzzy logic technology one usually also apply trapezoidal fuzzy set based semantics of words, whose cores are intervals, whereas the cores of triangular fuzzy sets are single value sets. This suggests us to think of the fact that the semantics of words, viewed as fuzzy information granules, may have their kernels. In the fuzzy set framework, the fuzzy set based semantics, including the trapezoidal fuzzy sets, are assigned intuitively by the human user to words. Of course, the word kernel still cannot be formalized in it. Whereas in the algebraic approach we will show that this concept can axiomatically be formalized in a reasonable way, which leads to the concept of enlarged hedge algebras (EnHAs). To model the kernel of any word $x \in X$, we need an additional artificial hedge, denoted by $h_{0}$, whose function is to generate the kernel of $x$. So, $h_{0} x$ is the kernel of $x$ and considered as representing the semantic core of words. The way to develop EnHAs is similar as the way for the ordinary HAs. The idea to develop the concept of EnHAs is as follows: Any given linear HA $\mathcal{A} X=\left(X, G, C, H_{I}, \leq\right)$ can be enlarged to become an EnHA $\mathcal{A} X_{\text {en }}=\left(X_{e n}, G, C, H_{e n}, \leq\right)$ by an addition of $h_{0}$ to $H_{I}$ and by introduction of the following axiom (A5) for simulating properties of the semantics core of words, $h_{0} x, x \in X$ (note that Axioms (A1) - (A4) can be found in [3]):

(A1) The unit operation $V$ (i.e. the greatest element) in $H^{+}$either is positive or is negative w.r.t. any operations in $H$. Particularly, $V$ is positive w.r.t. itself and the maximal operation $L$ in $H$.

(A2) If $u, v \in X$ are independent, i.e. $u \notin H(v)$ and $v \notin H(u)$, then $x \in H(u) \Rightarrow x \notin H(v)$.

(A3) Hedge inheritance $e^{2}$ : For $\forall x \in X, \forall h, k, h^{\prime}, k^{\prime} \in H$, we have

(i) $x \neq h x \Rightarrow x \notin H_{I}(h x)$.

(ii) $h \neq k \& h x \leq k x \Rightarrow h^{\prime} h x \leq k^{\prime} k x$.

(iii) If $h x \neq k x$, then $h x$ and $k x$ are independent.

(A4) For $\forall u \in X$, if $v \notin H_{I}(u)$ and $v \leq u(v \geq u)$, then $v \leq h u(v \geq h u)$, for every $h \in H_{I}$.

(A5) Axioms for the semantics core of words [10]: For $\forall x, y \in X_{e n}$ and $x \neq y$,

(i) $h^{\prime} h_{0} x=h_{0} x$, for $\forall h^{\prime} \in H_{e n}$, i.e. $h_{0} x$ is always a fixed point, and, for $x \in X, h_{0} x=x$ if and only if $x$ is constant, otherwise, $h_{0} x$ and $x$ are incomparable.

(ii) $\forall x, y \in X, x<y \Rightarrow h_{0} x<y \& x<h_{0} y$.

It is interesting that the statements (i) and (ii) are sufficient to describe the semantics cores of the words in $X$ : the kernels of the constants are just themselves; the kernel of a word must be included in it, but inclusion cannot be described in the order-based structures and, therefore, they should be incomparable. Nevertheless, it can be proved in the theory of EnHAs that the fuzziness intervals of the kernels of words should be included in the fuzziness intervals of their respective words.

The following properties of $\mathcal{A} X_{\mathrm{en}}$ describe the semantics core of the words and the orderbased structure of $X_{e n}$. First, we introduce some notations: for the underlying set $X$ of $\mathcal{A} X$ we put $X_{k}=\{x \in X:|x|=k\}$ and $X_{(k)}=\{x \in X:|x| \leq k\}$, while for $X_{e n}$ we have $X_{e n, k}=X_{k} \cup\left\{h_{0} u: u \in X_{(k-}\right.$ 1) $\}$ and $X_{e n,(k)}=\left\{x \in X_{e n}:|x| \leq k\right\}=X_{(k)} \cup\left\{h_{0} u: u \in X_{(k-1)}\right\}$.

\footnotetext{
${ }^{2}$ Suitably, this terminology is used instead of in our previous ones, hedge heredity, e.g. in Nguyen and Wechler, 1990
} 
Theorem 2.1. Let $\mathcal{A} X^{\mathrm{en}}=\left(X_{e n}, G, C, H_{e n}, \leq\right)$ be an EnHA of a free linear HA $\mathcal{A} X=(X, G, C, H$, $\leq)$. Then,

(i) $X_{e n}=X \cup\left\{h_{0} x: x \in X \backslash C\right\}$ and, for $x \notin C, h_{0} x \notin X$.

(ii) For $\forall x, y \in X_{e n}$, we have $x<y \Leftrightarrow x<h_{0} y \Leftrightarrow h_{0} x<y \Leftrightarrow h_{0} x<h_{0} y$. Consequently, $\left\{h_{0} x: x\right.$ $\in X\}$ is linearly ordered.

(iii) The set $X_{e n, k}=X_{k} \cup\left\{h_{0} u: u \in X_{(k-1)}\right\} \subseteq X_{e n}$ is also linearly ordered.

Since the concept of the fuzziness measure of words and its axiomatization are almost the same as in the case of ordinary HAs, except the need of introduction of the fuzziness measure of the constants, $f m(\boldsymbol{0}), f m(W)$ and $f m(\mathbf{1})$, which are possibly different from zero, and of the specific hedge $h_{0}, \mu\left(h_{0}\right)$, its axiomatization that can be referred to [10] is ignored here.

Then, given $f m$, the fuzziness intervals will be defined as follows:

Definition 2.1. Given a fuzziness measure of an EnHA $\mathcal{A} X_{\text {en }}$ of a linguistic variable $X$ and let us assume that each word $x \in X_{e n}$ of a linguistic variable $X$ is associated with an interval $\mathfrak{I}(x) \subseteq$ $[0,1]$, the normalization of the reference domain of $X$. These intervals are said to form a system of fuzziness intervals of the words of $\mathcal{A} X_{\text {en }}$, provided the following conditions hold:

(fi1) $|\mathfrak{I}(x)|=f m(x)$, for all $x \in X_{e n}$, where $|\mathfrak{I}(x)|$ denotes the length of the interval $\mathfrak{I}(x)$.

(fi2) The set $\left\{\mathfrak{I}(h x): h \in H_{e n}\right\}$ forms a (binary) partition of $\mathfrak{I}(x)$ and the order of its intervals is in accordance with the order of their associated words, i.e. $(\forall x, y \in\{h x: h \in$ $\left.\left.H_{e n}\right\}\right)(x \leq y \Rightarrow \mathfrak{I}(x) \leq \mathfrak{I}(y))$.

To point out the correctness of this definition and the structure of the fuzziness interval family we have the following.

Theorem 2.2. Definition 4.2 is correct, i.e. for a given fuzziness measure $f m$, there exist uniquely a system of intervals of the words of $\mathcal{A} X_{\text {en }}$ satisfying the conditions (fi1) and (fi2) in the sense that the fuzziness intervals of every word in any two such systems differ from each other only at their end points. In addition, putting $\mathbb{I}_{k}=\left\{\mathfrak{I}(y): y \in X_{e n, k}\right\}=\left\{\mathfrak{I}(x): x \in X_{k}\right\} \cup\left\{\mathfrak{I}\left(h_{0} x\right)\right.$ : $\left.x \in X_{(k-1)}\right\}$, the structure of the fuzziness intervals of $X$ have the following properties: $X$.

(fi3) For $y \in H_{e n}(x)$ and hence $y \neq x$, we have $\mathfrak{I}(y) \subseteq \mathfrak{I}(x)$. Especially, $\mathfrak{I}\left(h_{0} x\right) \subseteq \mathfrak{I}(x), \forall x \in$

(fi4) For every integer $k>0$, the intervals $\mathfrak{I}(x), x \in X_{e n, k}$, form a binary partition of $[0,1]$ and their order is in accordance with the order of their associated words. That is we have:

$$
\forall x, y \in X_{e n, k}: x \leq y \Rightarrow \mathfrak{I}(x) \leq \mathfrak{I}(y) \text {. }
$$

(fi5) For every $k, \mathbb{I}_{k}$ is topologically finer than $\mathbb{I}_{k-1}$, i.e. every fuzziness interval in $\mathbb{I}_{k}$ should be included in a certain fuzziness interval in $\mathbb{I}_{k-1}$.

(fi6) The set $\left\{\mathfrak{I}\left(h_{0} x\right): x \in X\right\}$ is linearly ordered and its order is in accordance with the order of their words. Moreover, it is dense in $[0,1]$ in terms of the ordinary topology.

The concept of SQMs is now replaced with the concept of interval-valued SQMs, whose values are taken in $\mathbb{P I}\left([0,1]\right.$. For short, for every word-set $Y \subseteq X_{\text {en }}$, we denote by $h_{0} Y$ the set $\left\{h_{0} x\right.$ $: x \in Y\}$.

Definition 2.2. Let $\mathcal{A} X_{\mathrm{en}}=\left(X_{e n}, G, C, H_{e n}, \leq\right)$ be an EnHA of a given ordinary hedge algebra $\mathcal{A} X$. An interval-valued mapping $f: X_{e n} \rightarrow \mathbb{P I}([0,1])$, whose interval-values are right-closed and left- 
open, except when their left end-point is 1 , is said to be an interval-valued quantifying mapping (IVQM), provided the following conditions hold:

(IVQM1) $f$ preserves the order of the words in $X$, i.e. it is a homomorphism of $\left(X_{e n}, \leq\right)$ in the category of the ordered sets. That is, $\left(\forall x, y \in X_{e n}\right)(x \leq y \Rightarrow f(x) \leq f(y))$

(IVQM2) $f$ preserves the functionality of the hedge $h_{0}$, i.e. $\left(\forall x \in X_{\text {en }}\right)\left(f\left(h_{0} x\right) \subseteq f(x)\right)$

(IVQM3) $\mathcal{C}\left(f\left(h_{0} X\right)\right)=[0,1]$, where $f(Y)=\{f(x): x \in Y\}, Y \subseteq X_{e n}$.

Axiom (IVQM3) ensures that the numeric interval semantics of the words of $X$ should approximate any numeric values in $[0,1]$. So, we see that the concept of the IVQMs is defined in a very natural way and their axioms are minimal constraints and, hence, it seems to be most general. Although such IVQMs are defined by general conditions related directly to the IVQM concept, it is expected that they should even then be in a strict relation with the fuzziness concepts, the fuzziness measure and the fuzziness intervals of words. If the answer is affirmative, it contributes to demonstrate the soundness of the algebraic approach to the semantics of words.

Theorem 2.3. Let $f$ be an IVQM of the EnHA $\mathcal{A} X_{\text {en }}$ of a free LHA $\mathcal{A} X$. Then, for every $x \in X_{e n}$,

(i) $\mathcal{C}\left(f\left(h_{0} H_{I}(x)\right)\right)=\mathcal{C}\left(f\left(H_{I}(x)\right)\right)=\mathcal{C}\left(f\left(H_{e n, I}(x)\right)\right) \in \mathbb{P} \mathbb{I}([0,1])$. Especially, for $x \in\left(X_{e n} \backslash X\right) \cup C$, we have $\mathcal{C}\left(f\left(H_{I}(x)\right)=\mathcal{C}(f(x))\right.$.

(ii) $f(x)=f\left(h_{0} x\right)$.

(iii) $f$ induces a function $f m_{f}: X_{e n} \rightarrow[0,1]$, defined by $f m_{f}(x)=\left|\mathcal{C}\left(f\left(H_{l}(x)\right)\right)\right|$, especially, $f m_{f}(x)$ $=\mid \mathcal{C}\left(f(x) \mid\right.$, for $x \in C \cup\left\{h_{0} x: x \in X\right\}$, which satisfies the first two axioms of the fuzziness measure of $\mathcal{A} X_{\mathrm{en}}$ and, therefore, it is called a semi-fuzziness measure of $\mathcal{A} X_{\mathrm{en}}$.

In the paper [10], we have shown that the EnHAs offer a formal basis in which the word semantics produces the trapezoidal fuzzy set based semantics of words and can be applied to solve classification problems even more effectively than the method examined in [2] using triangles.

To end this section, it is worth emphasizing that although both fuzzy sets and hedge algebras all deal with words as uncertainty linguistic information, only hedge algebras can handle immediately words and deal with their qualitative semantics. Especially, hedge algebras can establish a strict mathematical foundation based on an axiomatization manner for this. It is important that there are many basic and fundamental facts which support for this assertion, as discussed above.

Since the more fundamental a theory, the more soundness and effective applications of the theory can be achieved, we may hope that the HA theory can solve problems of different areas more effectively than the fuzzy set based counterpart methods.

\section{THE HA APPROACH TO COMPUTING WITH WORDS}

The terminology of Computing With Words (CWW) was used first in 1996 by Zadeh in his study [11] this concept has been taken form in that paper. Since then CWW has attracted many attention of the fuzzy community and has been intensively investigated, especially, it can find various interesting applications [1]. Nevertheless, as it is discussed above and pointed out in [9] that, since the fuzzy set theory does not originate immediately from the order-based semantics of words, there is a "formalized gap" between the words and their fuzzy set based semantics. This may cause some significant shortcomings in distinct approaches to CWW, as discussed next. 
An important area of application of CWW is the decision making in a linguistic data context, for which linguistic scales and mathematical representation of words should be examined. To limit these shortcomings of CWW in this area, the authors of [9] have argued that computing with words in a linguistic scale must be realized on word quantitative semantics and the words of the scale are used for human users expressing their assessments in decision making. They have also formulated explicitly two characteristics of linguistic scales for decision making:

"Property 1: Linguistic scales should have a semantic representation model with an explicit declaration of qualitative and quantitative semantics of the linguistic words of the scales. The qualitative semantics of terms is devoted to experts to express their linguistic assessments, while the quantitative semantics of terms is exploited to develop the computationally operational mechanism of the scales.

Property 2: Linguistic scales should be associated with their respective semantic linguistic scales constructed based on the proposed semantic representation model, which is equipped with an adequate computational structure so that it is closed with respect to necessary operations, including aggregation operators."

Then, in order to avoid the above mentioned shortcomings of existing approaches, they propose three requirements for dealing with linguistic scales and constructing a mathematical mechanism, called in that paper semantic linguistic scales, for computing with words of the linguistic scales:

"Requirement 1: Linguistic representation models of linguistic scales should be developed based on a clearly declared qualitative and quantitative semantics of linguistic words, which are related with their inherent order-based semantics as much as possible.

Requirement 2: There should be a suitable formalized mechanism based on the declared qualitative and quantitative semantics of words to construct a semantic linguistic scale characterized by Property 1 and Property 2 with obvious computational characteristics useful for practical applications.

Requirement 3: The semantic linguistic scale should bring necessary advantages to develop computational operations for developing decision-making methods, including aggregation operators, in particular. The constructed semantic linguistic scales should be closed with respect to the developed aggregation operators."

Based on these, it can be drawn that linguistic scales developed in the existing approaches are very difficult to satisfy well these requirements, since in Section 2.1 we argue that the qualitative semantics of words in natural languages should be defined based on order-based semantics of the domains of linguistic variables. For instance, since assigning words of a linguistic variable to fuzzy sets by a meaning mapping $M$, in general, $M$ does not preserve the order-based semantics of words, noting that ranking fuzzy sets is a difficult problems. Therefore, the approaches relying upon the extension principle of fuzzy sets [12] have no formal basis to link with the order based semantics of words. To establish such a formal basis, linguistic domains should be mathematically formalized.

For the symbolic approaches, e.g. [12 - 14], one tries to manipulate immediately the words of given linguistic scales utilizing their order in their scales. However, the quantitative semantics of words is not explicitly declared. So, the question is that on which quantitative semantics the developed operations on such scales act on? Analyzing how the proposed operations on the scales of symbolic approaches were defined we recognize that the indexes of the words in the scale of interest are utilized to define the computing mechanism of the scale. As it is discussed above, operations of the scales should work on the words semantics and, hence, the question is 
that may we declare explicitly that the quantitative semantics of the words in the given scales are their position indexes in the scales? It seems that it is impossible.

The HA approach provides a formal basis to develop computing mechanism for linguistic scales by discovering the formal structure of the linguistic scales and developing the respective semantic linguistic scales associated with them. This formal basis has the following interesting main features:

1) The semantics of a word present in a given linguistic scale is determined mainly in the context of the whole of its linguistic variable, i.e. it is defined in the free (infinite) HA $\mathcal{A} X$ associated with the variable. However, a scale is finite and the semantics of its words is changed a bit by the influence of their neighboring words in the scale. For example, the semantics of the word "good" of the variable QUALITY of a technology project appearing in between its neighboring words "medium" and "extremely good" of a scale is changed when it appears in between "rather good" and "very good" in another scale. That is, the specificity (vs. the generality) of a word can be changed, but certain specific key semantics of the word are still maintained, when its neighboring words are changed.

2) The structure of the linguistic scales determines or produces their respective semantic linguistic scales based on the structure of the variable hedge algebra. In other words, the qualitative semantics of the words of a given linguistic scale determines its computing structure of its semantic linguistic scale. This ensures, based on a formalized basis, that when someone deals with the semantic linguistic scale, its computing structure ensures that he still manipulates directly with its words to a certain extent.

Now, we describe how a given linguistic scale can determine its 4-tuple semantic linguistic scale based on the formal basis proposed in [9].

Let be given a linearly ordered linguistic scale $T=\left\{x_{j}: j=1, \ldots, n\right\} . T$ is said to superiorclosed provided that if $T$ contains a child $h x$, for some hedge $h$, then $T$ must also contain the word $x$ (words: strings of hedges and an atomic word). Denote by $x_{L}$ and $x_{R}$ respectively the left adjacent and the right adjacent of the word $x$ in the T-context (i.e. in the scale $T$ ). Remember that $X_{(p)}$ denotes the set of all words of length $\leq p$, where $p>0$ is an integer. Then, the following can be proved:

Proposition 3.1. Let $T$ be a superior-closed word-scale of $\mathcal{A} X$ with a specificity $l$ (the maximal length of the words in $T$ ). Then, for every $x \in T \backslash C, x_{L}$ is also the left adjacent word of $x$ in the $X_{\left(p_{L}\right)}$-context, where $p_{L}=\max \left(\left|x_{L}\right|,|x|\right) \leq l$ and $x_{R}$ is also the right adjacent word of $x$ in the $X_{\left(p_{R}\right)}$ context, where $\left.p_{R}=\max \left(\left|x_{R}\right|,|x|\right) \leq l\right)$. Particularly, if $x$ is of specificity $l$, i.e. $|x|=l$, then $x_{L}$ (respectively $x_{R}$ ) is also the left (respectively the right) adjacent term of $x$ in $X_{(l)}$.

This proposition asserts that we can determine the left (right) specificity degree indicated by $p_{L}\left(p_{R}\right)$ of the given word $x$ by calculating the index of $X_{\left(p_{L}\right)}\left(X_{\left(p_{R}\right)}\right)$. It is the basis to calculate the interval-semantics of $x$ using the similarity intervals of the terms in $X_{\left(p_{L}\right)}\left(X_{\left(p_{R}\right)}\right)$, noting that, for a given $k$, these intervals of the degree $k$ are only defined for the set $X_{(k)}$ :

Definition 3.1. Let be given the fuzziness parameter values of $\mathcal{A} X$ and $v$ is the SQM defined by these fuzziness parameters. Then, for every $x \in T$, the interval-semantics of $x$ in the context of $T$ is defined to be the interval $I(x)=I_{L}(x) \cup I_{R}(x)$, where $I_{L}(x)=L \varsigma_{p_{L}}(x)=\left[\operatorname{lpt} \varsigma_{p_{L}}(x), v(x)\right)$ with $p_{L}=$ 
$\max \left(\left|x_{L}\right|,|x|\right)$ and $I_{R}(x)=R \varsigma_{p_{R}}(x)=\left[v(x), r p t \varsigma_{p_{R}}(x)\right)$ with $p_{R}=\max \left(\left|x_{R}\right|,|x|\right)$ with $\varsigma_{p}(x)$ denoting the similarity interval of $x$ with degree $p$, i.e. $\varsigma_{p}(x)$ is defined for every $x \in X_{(p)}$.

Then, the 4-tuple semantic linguistic scale of the given linguistic scale $T$ is calculated by the following proposition:

Proposition 3.2. Let $S$ be a superior-closed linguistic scale with a specificity level $l$ of a given hedge algebra $\mathcal{A} X=(X, G, C, H, \leq)$. Then, for given fuzziness parameter values of $\mathcal{A} X$, the set $S_{v}$ $=\left\{\left(s, I_{\partial(s)}(s), v(s), r_{s}\right): s \in S, r_{s} \in I_{\partial(s)}(s)\right\}$ satisfies the following primary properties:

(i) $S_{v}$ is 4-tuple semantic linguistic scale associated with $S$.

(ii) Every interval $I_{\partial(s)}(s)$ is defined and calculated based on the semantics of the terms of $\mathcal{A} X: I_{\partial(s)}(s)=I_{L}(s) \cup I_{R}(s)$ and

$$
I_{\partial(s)}(s)==\bigcup\left\{\mathfrak{I}(x): x \in X_{l+2} \& \mathfrak{I}(x) \subseteq\left[v\left(s_{L, p_{L}+1}\right), v\left(s_{R, p_{R}+1}\right)\right)\right\} .
$$

To capture more details of this formal basis of the construction of semantic linguistic scales, the reader can refer to [9]. However, with the above presentation we can see that the construction examined in that paper is based on a very strict mathematical and logical (semantic) basis and, therefore, it is called sound construction of semantic linguistic scales.

To show the benefits of the HA approach to such a problem of CWW, a simple decision making problem is examined in [9]. Let us consider a decision making problem with two alternatives $A_{1}$ and $A_{2}$ and three criteria $C_{k}, k=1,2,3$. For simplicity, we assume that only one expert use the same linguistic scale for all three criteria to express the assessments of her/his evaluation of all the alternatives under consideration with respect to these distinct criteria. In addition, to make a clearly visible difference of the proposed approach from the 2-tuple based approach, two linguistic scales, the one is a proper subset of the other, that will be applied in turn are given as follows:

1) The scale

$S_{1}=\left\{s_{1, i}: i=1, \ldots, 9\right\}=\left\{E \_b a d, V \_b a d, b a d, R \_b a d\right.$, medium, $R \_g o o d$, good,$V \_g o o d$, Excellent $\}$.

2) The scale examined in Example 4.1 with

$S_{2}=\left\{s_{2, i}: i=1, \ldots, 5\right\}=\left\{\right.$ bad,$R \_b a d$, medium, good, Excellent $\}=S_{1} \backslash\left\{E \_b a d, V \_b a d\right.$, $R \_$good, $V \_$good $\}$, where, E_bad $\stackrel{\Xi}{=}$, Excellent $\triangleq 1$.

With the given independent fuzziness parameter values $\mu(V)=0.484$ and $f m\left(c^{-}\right)=0.5687$, the 4-tuple semantic linguistic scales associated with $S_{1}$ and $S_{2}$ are calculated and given as follows:

$S_{1}$ consists of the following 4-tuples:

$\left(E \_b .,[0,0.65), 0.31, r_{1}\right), \forall r_{1} \in I_{2}(\boldsymbol{0}) ; \quad\left(V_{-} b .,[0.65,2.07), 1.33, r_{2}\right), \forall r_{2} \in I_{2}\left(V_{-} b.\right) ;(b .$, $\left.[2.07,3.49), 2.75, r_{3}\right), \forall r_{3} \in I_{2}(b.) ;\left(R \_b .,[3.49,0.5), 4.27, r_{4}\right), \forall r_{4} \in I_{2}\left(R \_b.\right) ;(W,[0.5,6.21)$, 5.69, $\left.r_{5}\right), \forall r_{5} \in I_{2}(W) ;\left(R \_g .,[6.21,7.36), 6.77, r_{6}\right), \forall r_{6} \in I_{2}\left(R \_g.\right) ;\left(g .,[7.36,8.43), 7.91, r_{7}\right)$, $\forall r_{7} \in I_{2}(g.) ;\left(V \_g .,[8.43,9.51), 8.99, r_{8}\right), \forall r_{8} \in I_{2}\left(V_{-} g.\right) ;\left(\right.$ Excellent, [9.51, 1.0), 10.0, $\left.r_{9}\right), \forall r_{9} \in$ $I_{2}(1)$.

$S_{2}$ consists of the following 4-tuples: 
(b., [0, 3.49), 2.75, $\left.r_{1}\right), \forall r_{1} \in I_{1}(b.) ;\left(R \_b .,[3.49,0.5), 4.27, r_{2}\right), \forall r_{2} \in I_{2}\left(R \_b.\right) ;(W,[5.0$, 6.77), 5.69, $\left.r_{3}\right), \forall r_{3} \in I_{1}(W)$; (Good, [6.77, 8.99), 7.91, $\left.r_{4}\right), \forall r_{4} \in I_{1}(G$.$) ; (Excellent, [8.99, 10.0],$ $\left.10.0, r_{7}\right), \forall r_{7} \in I_{1}($ Excellent $)$.

Assume that the linguistic assessments of the two alternatives in question of the expert as shown in Table 3.1 can be considered as his assessments in the context of each of the two scales $S_{1}$ and $S_{2}$. Note that the weights of the criteria are also given in the table assuming that the selected aggregation operation is the weighted average.

Table 3.1 The evaluation provided by the expert with respect to the given criteria and their weights.

\begin{tabular}{|c|c|c|c|}
\hline Criteria and weights & $C_{1}, w_{1}=0,25$ & $C_{2}, w_{2}=0,51$ & $C_{3}, w_{3}=0,24$ \\
\hline$A_{1}$ & $s_{9}=$ Excellent & $s_{5}=$ medium & $s_{7}=$ good \\
\hline$A_{2}$ & $s_{4}=R \_$bad & $s_{9}=$ Excellent & $s_{4}=R \_$bad \\
\hline
\end{tabular}

As discussed in the first feature, their semantics of the expert's linguistic assessments given in Table 3.1 may be changed a bit by the influence of possible changes in their left and right adjacent words in each scale. However, as these assessments are in $S_{2} \subseteq S_{1}$, we have an intuitive basis to believe that, under this situation (the same word-assessments and $S_{2}$ is extended to $S_{1}$ ), the expert decision cannot be changed when $S_{2}$ is extended to $S_{1}$. As we expect, it has been shown in [9] that while his decision based on the 4-tuple semantic linguistic scale remains the same for both $S_{1}$ and $S_{2}\left(A_{1}\right.$ is more preferable than $\left.A_{2}\right)$, it is changed when linguistic 2-tuples are applied. This shows that the theory of hedge algebras seems to provide a reasonable and sound mathematical basis for CWW.

\section{APPLICATION IN SOLVING SOME CLASSIFICATION PROBLEMS USING FUZZY RULE BASED SYSTEMS}

A natural question is that when applying this algebraic approach to knowledge based systems, which novelties of methodologies and techniques it can bring out for enhancing the performance of knowledge based systems? Based on a fundamental formalized basis that the algebraic approach can provide, there are many advantages we may expect $[2,10]$ :

- The design of words: When regarding words as playing a centric role, similarly as the role of human-centric problem, it is for the first time words along with their fuzzy sets can concurrently be dealt with and, moreover, be integrated as a whole. This permits to design words for specific applications, noting that words are application-dependent. For example, the word "young" of age and "fast" of speed are application-dependent, as the meaning of "young" is different when they are used in the "world" of the only scientific staffs, or of the only scientific experts, or of the population of a state, and so on. Therefore, while words must be pre-specified in the fuzzy set based methodologies in many studies, in the HAapproach they are selected by learning strategies similarly as the way the human beings acquire their knowledge from reality. This would, of course, enhance the performance of fuzzy rule based systems (FRBSs).

- The generality and specificity of words: This allows develop methods that are able to simulate the interaction between words and real datasets (domain reality) as well as between linguistic rules and datasets. An emphasis should be made on the fact that the generality and specificity are significant characteristics of words for cognizing the realty. We will see in the 
sequel that there are sound techniques for dealing with these characteristics of words in the algebraic approach.

- Reducing complexity: In many existing methods in the literature of FRBSs, all possible combinations of word-values of dataset features are taken into account. Evidently, the number of all such rules is too huge in comparison with the cardinality of a given dataset. In the HA-approach we can avoid this problem, utilizing the similarity intervals of the words, which form a binary partition of their feature universe. Then, a feature-value of the given dataset falls into only a unique similarity interval of a certain word. Therefore, every pattern defines only one linguistic fuzzy rule, called basic rule. This decreases significantly the number of rules to be considered. We will point out that this technique offers meaningful role in solving problems.

- Knowledge interpretability: A crucial criterion to measure the interpretability of linguistic knowledge is to be intended as "user ability to read and understand" that mainly concerns "a comparison between the semantics of a knowledge base and the semantics of the knowledge acquired by a user after reading and understanding the knowledge base." When words appearing in the knowledge can be designed properly, as described above, they may be just what the user actually understands and, hence, the knowledge interpretability can be guaranteed.

With these advantages we expect that the HA-approach may ensure enhancing effectiveness in designing FRBSs, including fuzzy rule based classification systems (FRBCSs). The next simulation results illustrate this assertion.

\subsection{The design of fuzzy rule based classification systems using triangular fuzzy sets}

The problem is as follows: Consider a classification problem $\mathcal{P}$ given by a dataset $P=\left\{p_{l}=\right.$ $\left.\left(d_{l}, C_{l}\right): d_{l} \in D, C_{l} \in C, l=1, \ldots, N_{P}\right\}$, where $d_{l}=\left(d_{l, 1}, d_{l, 2}, \ldots, d_{l, n}\right) \in D$ of $n$ dimensions, $C=\left\{C_{l}\right.$ $: l=1, \ldots, M\}$ is the set of class names. Develop method based on Multi-Objective Optimization Using triangular fuzzy sets to solve $\mathcal{P}$ with high performance and low rule base complexity.

Because of limited space, we present here only the simulation results. For the method's details, refer to [2].

Table 4.1. Comparison of fuzzy rule base complexity using the Wilcoxon test at level $\alpha=0.05$

\begin{tabular}{|l|c|c|c|c|c|c|}
\hline \multicolumn{1}{|c|}{ VS } & $\mathrm{R}^{+}$ & $\mathrm{R}^{-}$ & Exact P-value & Asymp. P-value & Confidence interval & Exact Confidence \\
\hline All Granularities & 83.0 & 70.0 & $\geq 0.2$ & 0.740367 & {$[-52.4985,25.0426]$} & 0.95524 \\
\hline Prod./1-ALL & 153.0 & 00.0 & $1.5258 \mathrm{E}-5$ & 0.000267 & {$[-235.1573,-60.2954]$} & 0.95524 \\
\hline Prod./1-ALL TUN & 121.0 & 32.0 & 0.0348 & 0.033154 & {$[-29.4122,-0.5219]$} & 0.95524 \\
\hline
\end{tabular}

Table 4.2. Comparison of FRBCS performance using the Wilcoxon test at level $\alpha=0.05$

\begin{tabular}{|l|c|c|c|c|c|c|}
\hline \multicolumn{1}{|c|}{ VS } & $\mathrm{R}^{+}$ & $\mathrm{R}^{-}$ & Exact P-value & Asymp. P-value & Confidence interval & Exact Confidence \\
\hline All Granularities & 134.0 & 19.0 & 0.004638 & 0.006040 & {$[0.740583,3.436272]$} & 0.95524 \\
\hline Prod./1-ALL & 136.0 & 17.0 & 0.003158 & 0.004507 & {$[0.639143,3.117368]$} & 0.95524 \\
\hline Prod./1-ALL TUN & 121.0 & 32.0 & 0.034800 & 0.033154 & {$[0.116358,2.567368]$} & 0.95524 \\
\hline
\end{tabular}

The proposed method is applied to 17 classification datasets found in http://sci2s.ugr.es/keel/ category.php?cat=clas. Here, we exhibit the statistic comparison tests 
using the Wilcoxon test made on the simulation results of the datasets and analyze some benefits of the approach. The comparison results given in Table 4.1 show that the complexities of the fuzzy rule bases obtained by the proposed method are lower or more or less equal to the complexity of those obtained by the counterpart methods. Whereas, the statistic comparison results given in Table 4.2 show that the FRBCSs designed by the proposed method outperform the FRBCSs designed by other methods.

The question is how these advantages discussed above are exposed in this application. First, the words integrated with their triangles of all features can actually be designed and they are generated by the obtained optimal fuzziness parameters of the dataset features. For illustration, consider dataset Mammographic for which the optimized solution indicates that the maximal length $\mathrm{F}[j]$ of the words of the feature $j$, for $j=1$ to 5 , are found to be 3,2,3,2 and 2, respectively. The fuzziness measures of $c^{-}$of the five features are, respectively, 0.362608, $0.499927,0.519758,0.447016$ and 0.427377 . While, the fuzziness measures of the hedge $L$ (Little) of the features are $0.366572,0.529550$, $0.577176,0.655763$ and 0.320246 . They produce the designed words and their triangles, e.g. for the feature F[3], as exhibited in Fig.4.1.

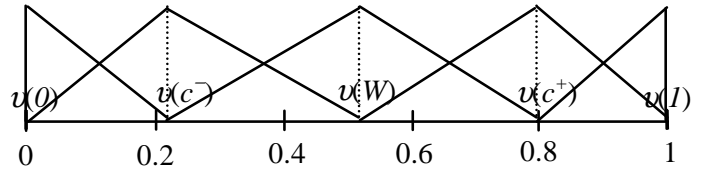

a) The fuzzy sets of the terms in $\boldsymbol{X}_{1}$

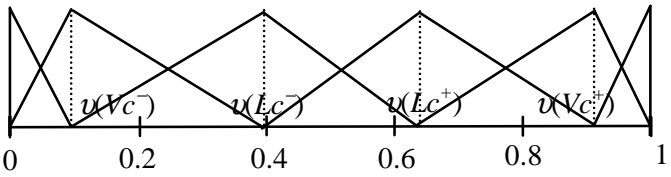

b) The fuzzy sets of the terms in $\boldsymbol{X}_{2}$

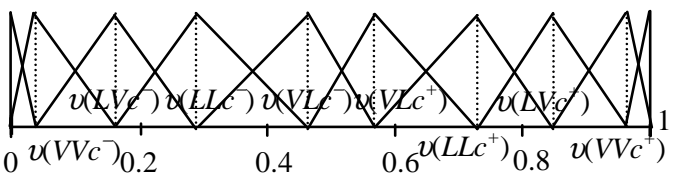

c) The fuzzy sets of the terms in $\boldsymbol{X}_{3}$

Figure 4.1. The fuzzy sets designed for the $3^{\text {th }}$ feature of the Mammographic dataset. As the maximal length of $F[3]$ is 3, i.e. the optimal solution points out that the words of the specificity of degree 3 are needed. We see that the fuzziness parameters obtained as above determine an appropriate "word stock" for each feature potentially used for formulating knowledge rules. In reality, which words are actually present in the rule base of a designed FRBCS depends strongly on the given dataset. In the fuzzy set framework, the size of the mentioned "word stock" is limited rather strictly and should be prespecified in many approaches, maybe because one has to consider all combinations of the feature linguistic values to generate the initial rules. However, it is not the case of the HA approach: we start with only the rules produced from the patterns of a given dataset, i.e. the number of such rules is not greater than the cardinality of the dataset. The "word stock" of potential words produced as above can be reasonably large that seems to be flexible, reasonable and compatible with the way human acquires their rules.

The "stock" of the designed words seems to meet the expected requirements. Although there

Table 4.2.a Frequencies of the occurrences of the designed linguistic values of all features in the 30 rule bases obtained by performing the 10-fold cross validation method for Mammographic dataset.

\begin{tabular}{|c|c|c|c|c|c|c|c|c|c|c|c|c|c|c|c|c|c|c|c|c|c|}
\hline & $\boldsymbol{0}_{-3}$ & $\boldsymbol{0}_{-2}$ & $\boldsymbol{0}_{-1}$ & $V V c^{-}$ & $V c^{-}$ & $L V c^{-}$ & $c^{-}$ & $L L c^{-}$ & $L c^{-}$ & $V L c^{-}$ & $W$ & $V L c^{+}$ & $L c^{+}$ & $L L c^{+}$ & $c^{+}$ & $L V c^{+}$ & $V c^{+}$ & $V V c^{+}$ & $\boldsymbol{1}_{-1}$ & $\boldsymbol{1}_{-2}$ & $\boldsymbol{1}_{-3}$ \\
\hline $\mathrm{F}[1]$ & & & & & & & & & & & & & 34 & & 0 & 8 & & 12 & 7 & 17 & \\
\hline $\mathrm{F}[2]$ & & 1 & & & & & & & 29 & & 11 & & 16 & & 36 & & 6 & & & 0 & \\
\hline $\mathrm{F}[3]$ & 1 & 5 & 3 & & & & & 6 & & & & & 7 & & 0 & & & & 6 & 27 & 20 \\
\hline $\mathrm{F}[4]$ & & 5 & 7 & & & & & & 1 & & & & 4 & & 28 & & & & 21 & 11 & \\
\hline $\mathrm{F}[5]$ & & & & & & & & & & & & & 0 & & 25 & & & & 3 & 0 & \\
\hline & 1 & 11 & 10 & 0 & 0 & 0 & 0 & 6 & 30 & 0 & 11 & 0 & 61 & 0 & 89 & 8 & 6 & 12 & 37 & 55 & 20
\end{tabular}


are until 30 rule bases produced by performing the 10 -fold cross validation method repeated three times on the dataset Mammographic, it is observed that a considerable number of the designed words of the "word stock" were not used to formulate the optimized rule bases as it can be observed in Table 4.2.a. Indeed, while the "word stock" of the potential words for the dataset has 70 words (two features of having words of length $\leq 3$ have $20 \times 2$ words and three features of having words of length $\leq 2$ have $10 \times 3$ words), there are only 28 words that are used to formulate the rules of the 30 rule bases, i.e. there are 42 unused words. This shows that which words necessarily selected from the "word stock" to extract optimal rule base are dependent mainly on the given dataset and that the genetic design of words for a given classification dataset actually plays a meaningful role in simulating the human process of drawing a rule-based knowledge from the real world: his natural language is viewed as a word stock and he tries to formulate his linguistic rules representing his knowledge while carefully selecting appropriate words in his word stock. However, an emphasis should necessarily be made on the fact that although 42 words are unused, they play still their meaningful role, as their presence does contribute to determine the necessary semantics of the words in the stock, noting that the word semantics are context-dependent as it can be observed in Figure 4.1.

Similarly, in the HA approach the generality-specificity of words, which depends on whether the word length is large or small, plays also meaningful role. For example, Tab.4.2.a demonstrates that, among the words present in the 30 rule bases, there are 147 occurrences of words of length 1 and 163 occurrences of words of length 2 and only 47 occurrences of words of length 3 . Note that the more generality of words present in a rule base, the smaller number of its rules. In contrast, the more specificity of the words present in a rule base, the more exact the designed fuzzy system can classify. This shows that the HA-based method can find a tradeoff between the general and the specific words selected from the word stock to represent the knowledge drawn from the dataset.

Note that, in accordance our knowledge, the benefits analyzed above cannot be observed in the existing approaches.

\subsection{The design of FRBCSs using trapezoidal fuzzy set based semantics of words}

In Section 2.4 we have presented the modelling the core of the word semantics, another advantage of the HA-approach in modeling different features of the inherent qualitative semantics of words. It is observed that words viewed as fuzzy information granules have naturally their kernels. In accordance to our knowledge, this concept has not formally been defined and examined in the fuzzy set framework. At the same time, we may imagine that this concept is not easy to define in this framework. Next, we will show moreover that it will be applied to generate trapezoidal fuzzy set based semantics of words and, then, applied to solve classification problems. Again, according to our knowledge, in general in this research field, the fuzzy sets of words are only assumed to be triangular fuzzy sets. One of obvious shortcomings of this fuzzy set shape is that the membership degrees of these fuzzy sets around their cores decrease very quickly. So, it is expected that trapezoidal fuzzy sets will provide another alternative to design FRBSs and even be better than triangular ones, where, for brief, the proposed method above is called Triangle-Method. 
Table 4.3. The simulation results of the Trapez-Md vs. the Triangle-Md using PSO algorithm.

\begin{tabular}{|c|c|c|c|c|c|c|c|c|c|c|c|c|c|c|}
\hline \multirow{2}{*}{ Dataset } & \multicolumn{5}{|c|}{ Trapezoid-method } & \multicolumn{5}{|c|}{ Triangle-method } & \multirow{2}{*}{ 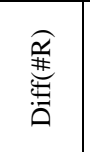 } & \multirow{2}{*}{ 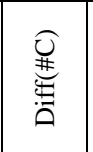 } & \multirow{2}{*}{ 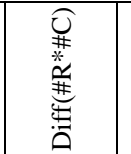 } & \multirow{2}{*}{ 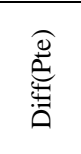 } \\
\hline & \#R & \#C & $\# \mathbf{R} * \# \mathbf{C}$ & Ptr & Pte & $\# \mathbf{R}$ & $\# \mathrm{C}$ & $\# \mathbf{R} * \# \mathbf{C}$ & Ptr & Pte & & & & \\
\hline Australian & 5.00 & 8.37 & 41.85 & 87.72 & 86.86 & 4.10 & 8.83 & 36.20 & 88.06 & 86.38 & 0.90 & -0.46 & 5.65 & 0.48 \\
\hline Bands & 7.00 & 11.17 & \begin{tabular}{|l|}
78.19 \\
\end{tabular} & 76.28 & 72.10 & 6.00 & 8.70 & 52.20 & 76.17 & 72.80 & 1.00 & 2.47 & 25.99 & -0.70 \\
\hline Bupa & 8.97 & 19.03 & 170.70 & 77.54 & 69.41 & 8.83 & 21.20 & 187.20 & 78.13 & 68.09 & \begin{tabular}{|l|}
0.14 \\
\end{tabular} & -2.17 & -16.50 & 1.32 \\
\hline Cleveland & 16.47 & 38.87 & & & 63.401 & 17.17 & 44.37 & 761.83 & 73.54 & 59.46 & -0.70 & -5.50 & -121.64 & 3.94 \\
\hline Dermatology & 10.87 & 17.43 & 189.46 & 96.88 & 95.521 & 10.90 & 18.17 & 198.05 & 98.03 & 96.07 & -0.03 & -0.74 & -8.59 & -0.55 \\
\hline Glass & 16.80 & 29.07 & 488.38 & 80.26 & 72.781 & 13.77 & 32.30 & 444.77 & 80.24 & 69.37 & 3.03 & -3.23 & 43.61 & 3.41 \\
\hline Haberman & 4.00 & 5.00 & 20.00 & 77.67 & 77.43 & 3.00 & 3.40 & 10.20 & 76.91 & 75.76 & 1.00 & 1.60 & 9.80 & 1.67 \\
\hline Heart & 8.03 & 15.03 & 120.69 & 88.07 & 84.57 & 7.67 & 16.10 & 123.49 & 89.45 & 84.20 & 0.36 & -1.07 & -2.80 & 0.37 \\
\hline Iono & 8.63 & 9.70 & 83.71 & 94.67 & 90.98 & 8.97 & 10.07 & 90.33 & 95.35 & 90.22 & -0.34 & -0.37 & -6.62 & 0.76 \\
\hline Mammogr. & 7.20 & 11.40 & 82.08 & 85.31 & 84.46 & 6.87 & 13.43 & 92.26 & 86.06 & 83.93 & 0.33 & -2.03 & -10.18 & 0.53 \\
\hline \begin{tabular}{|l|} 
Pima \\
\end{tabular} & 5.97 & 8.43 & 50.33 & 78.53 & 76.66 & 5.97 & 10.20 & 60.89 & 78.28 & 76.18 & 0.00 & -1.77 & -10.57 & 0.48 \\
\hline Saheart & 6.26 & 9.33 & 58.41 & 74.55 & 70.27 & 6.30 & 13.77 & 86.75 & 76.35 & 69.33 & -0.04 & -4.44 & -28.35 & 0.94 \\
\hline Sonar & 5.97 & 9.03 & 53.91 & 86.84 & 77.29 & 6.80 & 11.73 & 79.76 & 88.39 & 76.80 & -0.83 & -2.70 & -25.85 & 0.49 \\
\hline Vehicle & 11.03 & 19.60 & 216.19 & 71.64 & 68.121 & 11.60 & 20.77 & 240.93 & 70.54 & 67.30 & -0.57 & -1.17 & -24.74 & 0.82 \\
\hline Wdbc & 4.97 & 8.37 & 41.60 & 97.40 & 95.85 & 4.87 & 7.67 & 37.35 & 97.62 & 96.96 & 0.10 & 0.70 & 4.25 & -1.11 \\
\hline Wine & 5.87 & 7.17 & 42.09 & 1.00 & 98.52 & 5.57 & 6.43 & 35.82 & 99.88 & 98.30 & 0.30 & 0.74 & 6.27 & 0.22 \\
\hline Wisconsin & 6.93 & 8.30 & 57.52 & 96.74 & 96.45 & 6.93 & 10.73 & 74.36 & 97.81 & 96.74 & 0.00 & -2.43 & -16.84 & -0.29 \\
\hline
\end{tabular}

Table 4.4. Comparison of rule base complexity using the Wilcoxon test at level $\alpha=0.1$ for Trapez-Md.

\begin{tabular}{|c|c|c|c|c|c|c|}
\hline VS & $\mathrm{R}^{+}$ & $\mathrm{R}^{-}$ & Exact P-value & Asymp. P-value & Confidence interval & Exact Confidence \\
\hline Triangle PSO-Md & 107.0 & 46.00 & 0.15938 & 0.142245 & {$[-16.2359,1.42545]$} & 0.90162 \\
\hline
\end{tabular}

Similarly as above, we emphasize that in the HA-approach we can develop methods to produce algorithmically trapezoidal semantics of words based from given fuzziness parameter values. Since we can apply the same method of the FRBCS's design used in Section 4.1, we have a formal basis to show the meaningful role of the design of words based on the EnHAs presented in Section 2.4. To deal with this question, assume that we use the same method for the design of FRBCSs, except that words with trapezoidal fuzzy sets will be designed instead of the triangular ones. The new method is called Trapezoid-Method. In addition, if the same evolutionary algorithm is applied and the same number of generations for running the algorithms is specified, we are in position to ensure that only the word design factor can influent on the possible differences of the simulation results between the examined methods. Thus, the both methods are run with the use of the same PSO (Particle Swarm Optimization) algorithm and the same number of the generations which is specified by 1000 . The obtained simulation results of the both methods are presented in Table 4.3. At first glance we may conclude that while the rule base complexity measured by the $\operatorname{Diff}\left(\# \mathrm{R}^{*} \# \mathrm{C}\right)$ of almost datasets are negative, i.e. the complexity of the FRBCSs designed by the Trapezoid-Method has a tendency to be less than the one of the FRBCSs designed by the Triangle-Method, there are only 4 datasets for which the performance of the former systems is less than the one of the latter systems. Statistically, the Wilcoxon test results given in Table 4.4 and 4.5 also confirm these conclusions. As discussed above, this shows that the only factor that makes the Trapezoid-Method better than the TriangleMethod is the use of the trapezoidal fuzzy set based semantics of words. 
Table 4.5 Comparison of FRBCS performance using the Wilcoxon test at level $\alpha=0.05$ for Trapezoid-Method.

\begin{tabular}{|c|c|c|c|c|c|}
\hline $\mathrm{R}^{+}$ & $\mathrm{R}^{-}$ & Exact P-value & Asymp. P-value & Confidence interval & Exact Confidence \\
\hline 121.0 & 32.0 & 0.0348 & 0.033154 & {$[-17.65545,4.9465]$} & 0.95524 \\
\hline
\end{tabular}

Since in [2] it is demonstrated that the Triangle-Method is better than the counterpart fuzzy set based methods, these results confirm the meaningful role of the design of words with the trapezoidal fuzzy set based semantics and, hence, the practical value of the HA-approach [10].

\subsection{The design of hedge algebra based controllers}

Analyzing single-conditional fuzzy linguistic rule in natural language, we have a feeling that human beings formulate their fuzzy rule based control knowledge acquired from the reality based on their discovering direct or inverse proportional relations between physical variables. For example, the relation between two variables electric intensity $I$ and the seed $S P$ of an electrical motor can be formulated as "If $I$ is small then $S P$ is large", which is at least deduced from the inverse proportional relation between two numeric physical variables "intensity" and "speed" observed by the user. That is the order-based semantics of words is essential for representing human rule based knowledge. This implies that any mathematical model representing such knowledge must preserve these semantic order relations of linguistic variables. In the case of multiple-conditional fuzzy linguistic rules, the relation between two variables is much more complicated, however, every rule is formulated based on such relations above between every two variables.

Control knowledge is expressed by the following set of fuzzy linguistic rules:

$$
\text { If } X_{1} \text { is } A_{i 1} \text { and } \ldots \text { and } X_{m} \text { is } A_{i m} \text { then } \Upsilon \text { is } B_{i}, i=1, \ldots, n
$$

These rules describe dependencies between linguistic variables $X_{j}, j=1, \ldots, m$, and $\Upsilon$, where $A_{i j}$, $j=1, \ldots, m$, and $B_{i}$ are words of the linguistic variables $X_{j}$ and $\mathcal{Y}$, respectively, for $i=1, \ldots, n$.

HAs have found some applications to solve efficiently some control problems published in $[15-18,19,20]$. Although they are not many, but the significant thing seems that this efficiency comes just from the soundness of the HA-approach. In this section, we explain more obviously why we assert that the HA-approach to this field is sound and, for an additional illustration, a new result will be presented shortly to expose an additional benefit of the HA-approach. In [19, 20] we have pointed out several weak points of the fuzzy set based approach to solve control problems. Here, in order to show fundamental advantages of HA-approach we summarize main components, considered as hard problems, that influence the effectiveness of a general controller in the fuzzy set framework:

- Membership problem: To design the semantics of words of linguistic variables present in (3.1), which are represented by fuzzy sets designed in many ways and assigned to words by the designer. The parameters for defining the designed fuzzy sets are many since these fuzzy sets are in general designed independently from each other.

- Implication operator problem: To represent every fuzzy rule $r_{i}$ of (3.1) as a fuzzy relation $R_{i}(\boldsymbol{x}, y), i=1$ to $n$, where $\boldsymbol{x}$ is an $m$-vector, utilizing an $t$-norm or $t$-conorm to aggregate $m$ conditions of the rule and an implication operator $u \rightarrow v, u, v \in[0,1]$, to model the if-then semantics. 
- Aggregation problem: To aggregate also the obtained relations $R_{i}$ to produce one relation $R$, which can be considered as mathematical model of the control knowledge given by (3.1).

- Composition inference rule problem. To define a composition inference rule based on the following scheme: for an input $\boldsymbol{x}_{0}$, compute the output (control action) $y_{0}$ as follows:

(i) $B_{0}=\boldsymbol{A}\left(\boldsymbol{x}_{0}\right){ }^{\circ} R$; and (ii) $y_{0}=\operatorname{defuz}\left(B_{0}\right)$, where $\boldsymbol{A}\left(\boldsymbol{x}_{0}\right)$ is a fuzzy set obtained from $\boldsymbol{x}_{0}$ by a fuzzification method, ${ }^{\circ}$ is a selected composition and defuz is a defuzzification method.

We see that such a method depending on several well-known hard problems mentioned above seems to be so complicated that it may make the method to become a black box, i.e. it is difficult to recognize the behavior of the method to improve it. More importantly, the mappings of words to fuzzy sets and control methods described above do not preserve order-based structure of the linguistic fuzzy control knowledge. This weak point seems to be very fundamental on the mathematical and logical viewpoint and it may make the effect of these methods decreased.

In the HA-approach the general method is very simple. However, we first discuss about the soundness of the mathematical foundation for the proposed method.

The soundness of the HA-approach originates from two main facts. The first one is the order-based nature of linguistic knowledge, as discussed at the beginning of the section. The second one is that HAs model properly the order-based semantics of the words of variables. The order-based semantics of words appearing in human knowledge seems to be crucial and valuable, but it was ignored in almost studies of this field. For example, given a well-known rule saying that "if body temperature is very high then it is serious", we may imply that "if body temperature is extremely high then it is very serious". That is a proportional relation between the variables TEMPERATURE and HEALTH_STATUS in terms of the order relation on the linguistic domains appears.

Fortunately, hedge algebras model the order-based semantics of words and SQMs are isomorphisms in the category of order-based structures. Based on this, the following reasoning method was proposed:

- Consider every rule $r_{i}$ of (3.1) as defining a linguistic point $\left(A_{i 1}, \ldots, A_{i m}, B_{i}\right)$. Hence, the rules in (3.1) determine approximately a linguistic surface $S_{L}$. Note that the shape of $S_{L}$ depends on the order relationships between the words of and between the variables present in (3.1).

- Define suitable hedge algebras of the variables present in (3.1) and specify fuzziness parameter values of each variable. Then, the SQMs, $\mathfrak{v}_{\chi j}$, of the variables are fully defined (Section 2).

- Using $\mathfrak{b}_{x j}, j=1, \ldots, m$, transform $S_{L}$ into a Numeric surface $S_{N}$.

- To select an interpolation and extrapolation method on $S_{N}$.

It is very simple because the determination of HA for every variable is very easy, since its words are almost identical with words in natural (English) language. In addition, in practice of fuzzy control, only two hedges are sufficient. The number of the independent fuzziness parameters is very small, only two. It is important that they are the parameters of the whole variable, irrespective how many words are present in the control knowledge. When specifying values of these parameters, all the quantification characteristics of HAs, including their SQMs, are fully defined and calculated. In addition, the interpolation and extrapolation are familiar for any ones. Now, since there are only few numeric interpolation methods, with the simplicity above analyzed, it is interesting that the only difficult thing to be determine is the independent 
fuzziness parameter values, which, however, can feasibly be determined by trial-error, or even by an evolutionary algorithm [19].

It is most essential, however, that in modeling the mathematical model should preserve the math-structure of words of interest. Since $S_{N}$ is the isomorphic image of $S_{L}$ and the shape of $S_{N}$ is similar to $S_{L}$, we have a formal basis to believe that the interpolation on $S_{N}$ will produce appropriate control action values. All of these explain why we regard the proposed HA-based method as being sound. It is maybe by this reason the initial studies based on this method in this field can achieve more effective results in comparison with counterpart ordinary fuzzy control methods $[15-18,19,20]$.

To show further that a sound method will bring out the effectiveness in applications, we present below some plots describing the control effect of hedge algebra based controllers (HACs). The design of HACs comprises the following tasks:

- Determine $\mathcal{A} X_{j}=\left(X_{j}, G_{j}, C_{j}, H_{j}, \leq_{j}\right)$ for every linguistic variables $X_{j}$ present in fuzzy model (4.1). In recent practice, it is sufficient to use two hedges for each $H_{j}$, denoted by $L_{j}$ and $V_{j}$;

- Determine the fuzzy model using words in terms of elements the determined HAs $\mathcal{A} X_{j}$, as, usually, words present in (4.1) are of the

Table 4.6. The system parameters with ATMD.

\begin{tabular}{|c|c|c|c|}
\hline Storey $i$ & $\begin{array}{c}\text { Mass } m_{i} \\
\left(10^{3} \mathrm{~kg}\right)\end{array}$ & $\begin{array}{c}\text { Damping } c_{i} \\
\left(10^{2} \mathrm{Ns} / \mathrm{m}\right)\end{array}$ & $\begin{array}{c}\text { Stiffness } k_{i} \\
\left(10^{5} \mathrm{~N} / \mathrm{m}\right)\end{array}$ \\
\hline 1 & 450 & 261.7 & 180.5 \\
\hline $2-15$ & 345.6 & 2937 & 3404 \\
\hline 16 & $1 \mathrm{n} 4 \mathrm{918}$ & 5970 & 780 \\
\hline
\end{tabular}
form, for instance, "Negtive Big" $(N B)$ or "Positive Small" $(P S), \ldots$ This task can be realized by establishing the word-transformation that maps the words of in (4.1) into suitable words of the determined HAs. To preserve the semantics of words, all the established transformations should preserve the order-based relationships and the opposite meaning of terms, e.g. the opposite terms $N B$ and $P B$ are transformed respectively into $V_{j} S$ and $V_{j} B$, which are of opposite meaning in their respective HAs.

- Determine appropriate semantics of words of each $\mathcal{A} X_{j}$ by searching the independent fuzziness parameter values of $X_{j}$, the values of $f m\left(c_{j}^{-}\right)$and $\mu\left(L_{j}\right)$, for every $X_{j}$.

- Calculate the grid of points that define approximately the surface $S_{L}$ and determine an interpolative method on $S_{L}$.

Table 4.7. Rule base for the actuator on the $1^{\text {st }}$-storey.

\begin{tabular}{|c|c|c|c}
\hline$\dot{x}_{2}$ & $\mathrm{~N}$ & $\mathrm{Z}$ & $\mathrm{P}$ \\
\hline $\mathrm{NB}$ & $\mathrm{NB}$ & $\mathrm{NM}$ & $\mathrm{NS}$ \\
\hline $\mathrm{NS}$ & $\mathrm{NM}$ & $\mathrm{NS}$ & $\mathrm{Z}$ \\
\hline $\mathrm{Z}$ & $\mathrm{NS}$ & $\mathrm{Z}$ & $\mathrm{PS}$ \\
\hline $\mathrm{PS}$ & $\mathrm{Z}$ & $\mathrm{PS}$ & $\mathrm{PM}$ \\
\hline $\mathrm{PB}$ & $\mathrm{PS}$ & $\mathrm{PM}$ & $\mathrm{PB}$ \\
\hline
\end{tabular}

Table 4.8. Rule base for the actuator on the $15^{\text {th }}$-storey.

\begin{tabular}{|c|c|c|c|}
\hline$\dot{x}_{15}$ & $\mathrm{~N}$ & $\mathrm{Z}$ & $\mathrm{P}$ \\
\hline $\mathrm{NB}$ & $\mathrm{NB}$ & $\mathrm{NM}$ & $\mathrm{NS}$ \\
\hline $\mathrm{NS}$ & $\mathrm{NM}$ & $\mathrm{NS}$ & $\mathrm{Z}$ \\
\hline $\mathrm{Z}$ & $\mathrm{NS}$ & $\mathrm{Z}$ & $\mathrm{PS}$ \\
\hline $\mathrm{PS}$ & $\mathrm{Z}$ & $\mathrm{PS}$ & $\mathrm{PM}$ \\
\hline $\mathrm{PB}$ & PS & PM & PB \\
\hline
\end{tabular}

For illustration, we present some results of the application of the design of HACs and opHACs to a vibration problem of the control of high-rise structural systems presented in Figure 4.2 with active tuned mass damper (ATMD) against earthquakes to show the

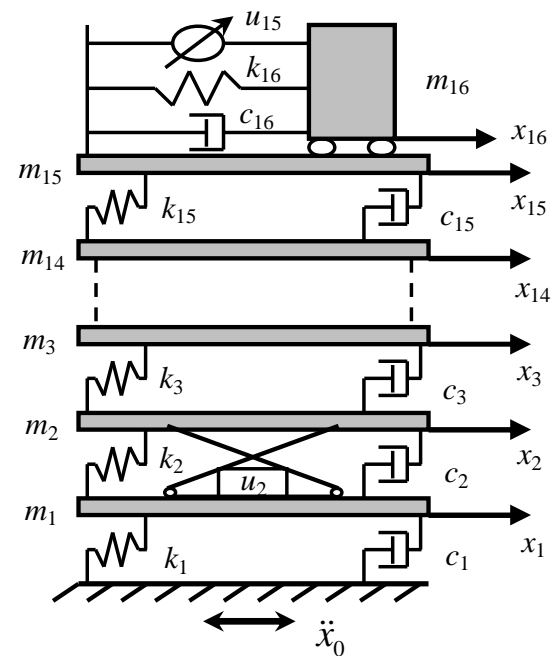

Figure 4.2. The structural system. 
advantages of the proposed HA methodology. These controllers were examined and simulated with the recorded seismic data of three typical earthquakes, El Centro, Northridge and Kobe, to demonstrate their performance and, by this, to contribute to state the advantages of the approach. A high-rise building structural system with ATMD assumed to have fifteen degrees of freedom all in a horizontal direction described in Figure 4.2, was taken into account to make a comparison study of distinct controllers.

Note that the fuzzy controllers (FCs) examined here were designed by the same method examined in [21].

1) Determining the control problem and its discrete control model: As it can be seen in Figure 4.2, the system is modeled with two active actuators of different types to suppress structural vibrations against earthquakes. Accordingly, one is installed on the first storey and the other on the fifteenth storey, since the maximum inter-storey shear force occurs on the first storey and the maximum displacements and accelerations are expected from the top storey of the structure during an earthquake, assuming equivalent storey stiffness and ultimate capacities. In Figure 4.2, $m_{1}$ is a movable mass of the ground storey and $m_{2}, m_{3}, \ldots, m_{15}$ are the masses of the remaining storeys, where the mass of all storeys include both the ones of storeys and their walls. The mass $m_{16}$ is of the ATMD installed on the fifteenth storey. The variables $x_{1}, x_{2}, x_{3}, \ldots, x_{14}$ and $x_{15}$ indicate the horizontal displacements and $x_{16}$ indicates the displacement of the ATMD. The variable $x_{0}$ is the earthquake-induced ground motion disturbance to the considered structural system. All springs and dampers are acting in the horizontal direction. The system and ATMD parameters examined in [21] are given in Table 4.6, which are used here for a comparative study.

Table 4.9. Linguistic transformation for

\begin{tabular}{|c|c|c|c|c|}
\hline \multicolumn{5}{|c|}{$x_{2}, \dot{x}_{2}, x_{15}$ and $\dot{x}_{15}}$. \\
\hline NB & $\mathrm{N}$ & $\mathrm{Z}$ & $\mathrm{P}$ & PB \\
\hline small & $\begin{array}{l}\text { Little } \\
\text { small }\end{array}$ & $\boldsymbol{W}$ & $\begin{array}{l}\text { Little } \\
\text { large }\end{array}$ & large \\
\hline
\end{tabular}

Table 4.10. Linguistic transformation for $u_{2}$ and $u_{15}$.

\begin{tabular}{|c|c|c|c|c|c|c|}
\hline NVB & $\mathrm{NB}$ & $\mathrm{N}$ & $\mathrm{Z}$ & $\mathrm{P}$ & $\mathrm{PB}$ & $\mathrm{PVB}$ \\
\hline $\begin{array}{c}\text { Very } \\
\text { small }\end{array}$ & small & $\begin{array}{l}\text { Little } \\
\text { small }\end{array}$ & $\mathrm{W}$ & $\begin{array}{l}\text { Little } \\
\text { large }\end{array}$ & large & $\begin{array}{l}\text { Very } \\
\text { large }\end{array}$ \\
\hline
\end{tabular}

Based on the discrete control model established based on the dynamic model of fifteendegrees-of-freedom structural system equipped with ATMD given in [21], the fuzzy rule bases of the two active actuators that were examined in that paper are given in Tab. 4.7 and 4.8.

2) Constructing control algorithm for the desired HAC: As discussed at the beginning of Section 4.3, the HA-rule base can be obtained by the selection of appropriate wordtransformations, which are given in Tab. 4.9 and 4.10.

- The design of HACs: The semantics of words of HACs were designed independently from the recorded seismic data of the three earthquakes mentioned above, i.e. not based on the semantics of words used in the common reality of earthquakes. In this situation, for all linguistic variables, we should have $\mu(L)=\mu\left(h_{-1}\right)=\mu(V)=\mu\left(h_{1}\right)=0.5, \mathrm{fm}($ small $)=0.5 ;$ fm $($ large $)=1-$ $f m($ small $)=0.5$. Even though, by simulation results, it will be seen that such HACs still work better than the counterpart standard FCs in controlling the system against earthquakes.

- $\quad$ The design of optimal HACs (opHAC): The fuzziness parameters for determining the semantics of words used in the context of earthquake data were optimized using the seismic data of El Centro earthquake in USA given at http://www. vibrationdata.com/elcentro.htm, which were recorded at the El Centro Terminal Substation Building on May 18th, 1940 with Peak 
Ground Acceleration (PGA) $0.35 g$, will be used for the design of $o p H A C s$. The idea of solving the fuzziness parameter optimization problem is described as follows: since it is difficult for the designer to determine the appropriate fuzziness parameters for a practical application problem, the data of El Centro earthquake is chosen randomly among three mentioned earthquakes as the training data to determine the near optimal fuzziness parameters for the earthquake protective structural system under consideration. They are regarded as the word semantics used for describing the seismic data in the reality of earthquakes. The goal function of the fuzziness parameter optimization problem is defined as follows:

$$
g=w_{1} \cdot g_{1}+w_{2} \cdot g_{2}+w_{3} \cdot g_{3} \text {, with } g_{1}=\sum_{j=0}^{n} \frac{x_{2}^{2}(j)}{a_{2}^{2}}, g_{2}=\sum_{j=0}^{n} \frac{x_{15}^{2}(j)}{a_{15}^{2}} \text { and } g_{2}=\sum_{j=0}^{n} \frac{x_{16}^{2}(j)}{a_{16}^{2}}
$$

where $x_{i}$ indicates the horizontal displacement of the i-th storey, $a_{i}$ indicates the absolute peak displacement, for $i=1, \ldots, 15$, and velocity vectors of the uncontrolled state of the structure excited by earthquake ground shaking; $x_{16}$ indicates the displacement of the ATMD; $n$ is the number of control cycles, the $a_{i}$ 's are specified above; and the positive weights $w_{1}, w_{2}$ and $w_{3}$ satisfy the equality $w_{1}+w_{2}+w_{3}=1$. The values of the weights should be carefully selected in the design of $o p \mathrm{HACs}$ for the application.

\begin{tabular}{|c|c|c|c|c|c|c|}
\hline$w_{1}$ & $w_{2}$ & $w_{3}$ & $\begin{array}{c}f m\left(c^{-}\right) \\
\left(V_{2}\right)\end{array}$ & $\begin{array}{c}\mu\left(h^{-}\right) \\
\left(V_{2}\right)\end{array}$ & $\begin{array}{c}f m\left(c^{-}\right) \\
\left(V_{15}\right)\end{array}$ & $\begin{array}{c}\mu\left(h^{-}\right) \\
\left(V_{15}\right)\end{array}$ \\
\hline 0.40 & 0.40 & 0.20 & 0.594037 & 0.500196 & 0.516618 & 0.543988 \\
\hline
\end{tabular}

For simplification of the evolutionary algorithm, only the semantics of the words of the variables $X_{2}$ and $X_{15}$ are optimized and the weights $w_{1}, w_{2}$ and $w_{3}$ are determined by trial-error. For the variable $U$ (control action $u$ ), its fuzziness parameters are defined as follows: $f m($ small $)=$ $\mu($ Little $)=0.5$. Then, the optimal fuzziness parameter values of $X_{2}$ and $X_{15}$ and the weight values were found, as follows.

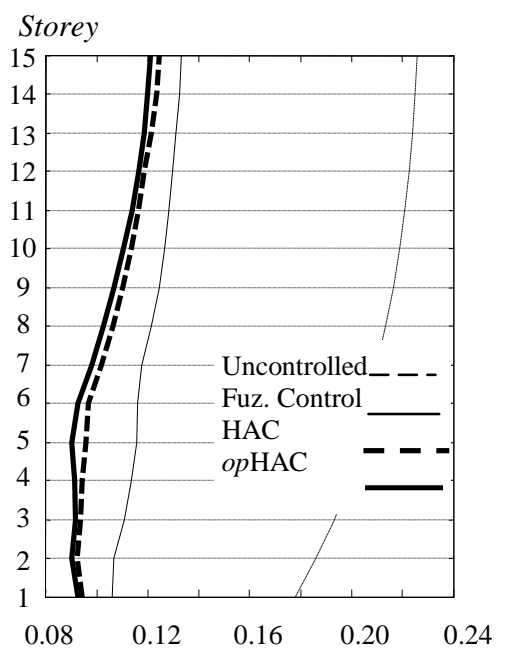

Figure 4.3. Peak Storey displacements (m), El Centro Earthquake.
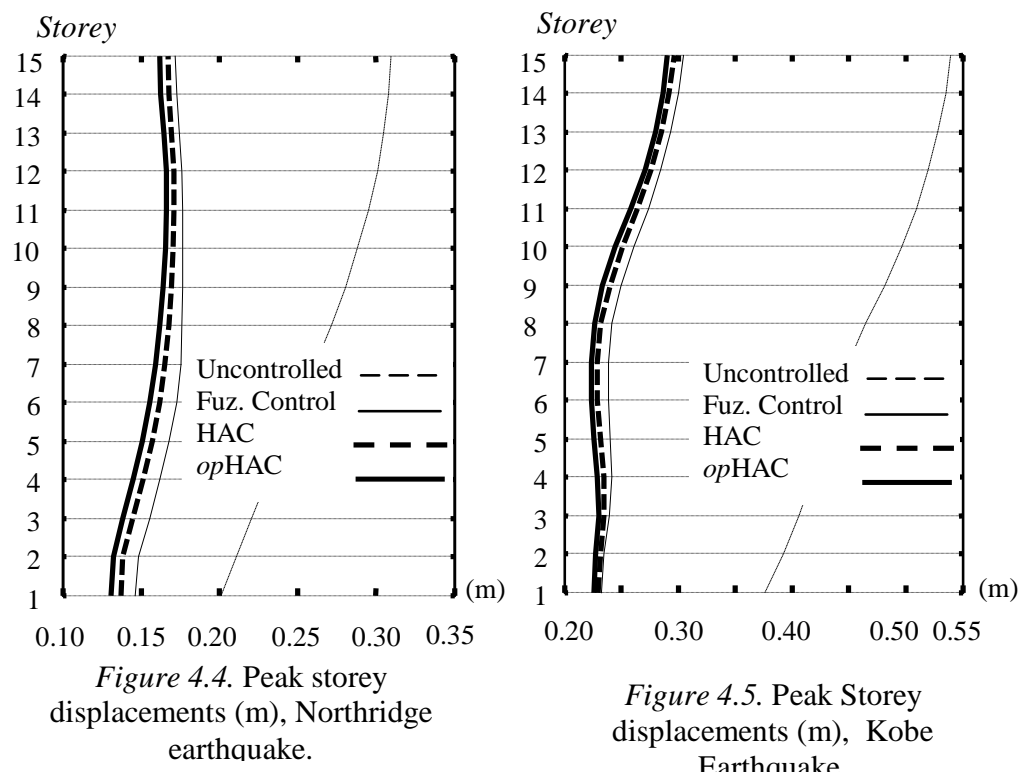

Figure 4.5. Peak Storey displacements (m), Kobe Earthauake. 


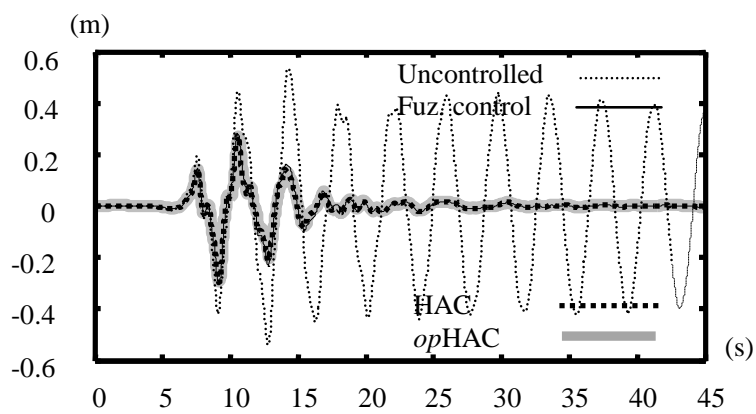

Figure 4.6. The time displacements responses of the top storey $\left(x_{15}\right)$ of Kobe earthquake.

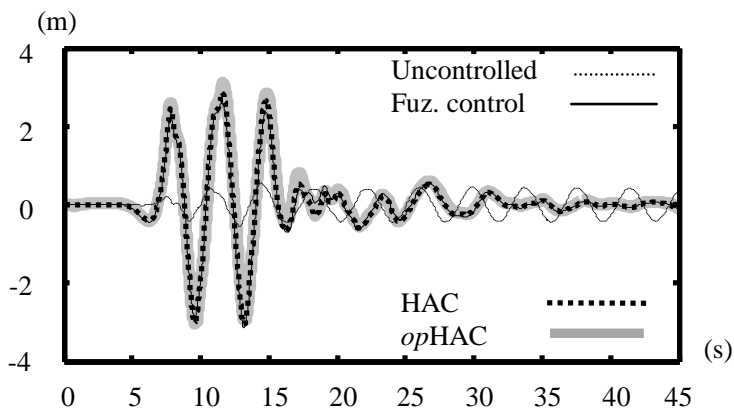

Figure 4.7. The time displacement responses of $\operatorname{ATMD}\left(x_{16}\right)$ of Kobe earthquake.

To see how well work the designed HACs and $o p H A C s$ in comparison with the standard designed FC, for reducing space of the report, we quote here only few plots of the simulation results studied in [22]:

(i) The displacement response: Figures 4.3 - 4.5 represent the peak displacements of all storeys, which indicate that the peak displacements produced by the designed controllers are increased from $\mathrm{FC}$ to $\mathrm{HAC}$ and then to $o p \mathrm{HAC}$ for all fifteen storeys of the building and in all three examined earthquakes.

(ii) The time responses of the displacements of only the top storey $\left(x_{15}\right)$ and the ATMD $\left(x_{16}\right)$ for the three controllers are depicted in Figures 4.6 and 4.7, respectively.

\section{CONCLUSIONS}

We have argued that HAs seems to be a sound mathematical structure for modelling and handling immediately the semantics of words. This assertion can be drawn from fundamental mathematical, logical and practical bases. On logical viewpoint of semantics of words, as syntactic expressions, the semantics of words should point at some things in reality. That is one has to think of at which items in reality a vague linguistic value like "beautiful" points at when a person uses this word. We have argued that he does not think of a "fuzzy set" of certain beautiful items. Stemming from the demand of human decision making we have pointed out that the word "beautiful" a human being uses aims to make a comparison between properties of certain items in the reality. This viewpoint seems to be much clearer if, for instance, we put this word in a context of words that includes "more beautiful", "very beautiful" and "rather beautiful".

On the practical viewpoint, it is natural that human beings handle immediately their words in their daily lives. Therefore, any theory that aims to simulate human capabilities should provides a sufficient mathematical formalism to deal immediately with words and their semantics that human being assign to them in reality. It can be observed that word-domains of linguistic variables can be viewed as order-based structures induced by the natural qualitative semantics of words. Therefore, HAs can be considered as a natural formalism for modeling the semantics of words. We show also that HAs are the formalized theory that deal directly with the inherent qualitative semantics. According to our knowledge, up to now only hedge algebras satisfy these requirements.

In addition, as we have presented in the report, they have been developed based on a strict axiomatic foundation, as their name "algebra" says. Remember that all concepts "fuzziness", 
"fuzziness measure" and "semantically quantifying mappings" are developed based on an axiomatization manner.

It offers many theoretical and methodological advantages and, hence, we may expect that it could bring out effective applications in different areas. The effectiveness of the initial applications of HAs in some distinct fields presented in this report contribute to realize this hope.

Acknowledgements. The research is funded by Vietnam National Foundation for Science and Technology Development (NAFOSTED) under Grant Number 102.05-2013.34.

\section{REFERENCES}

1. Mendel J. M. et. al. - What computing with words means to me, IEEE Comput. Intell. Mag. 5 (2010) (1) 20-26.

2. Thang Long Duong, Cat Ho Nguyen, Pedrycz W, Thai Son Tran - A Genetic Design of Linguistic Terms for Fuzzy Rule Based Classifiers, Int. J. Approx. Reason. 54 (2013) 0121.

3. Cat Ho Nguyen, Wechler W. - Hedge algebras: An algebraic approach to structures of sets of linguistic domains of linguistic truth variable, Fuzzy Set and Syst. 35 (3) (1990) 281293.

4. Cat Ho Nguyen, Wechler W. - Extended hedge algebras and their application to Fuzzy logic, Fuzzy Set and Syst. 52 (1992) 259-281.

5. Cat Ho Nguyen - A topological completion of refined hedge algebras and a model of fuzziness of linguistic terms and hedges, Fuzzy Set and Syst. 158 (2007) 436-451.

6. Cat Ho Nguyen, Van Long Nguyen - Fuzziness measure on complete hedge algebras and quantifying semantics of terms in linear hedge algebras, Fuzzy Set and Syst.1 58 (2007) $452-471$.

7. 13/ Nguyen Cat Ho - A Topological Completion of Refined Hedge Algebras and a Model of Fuzziness of Linguistic Terms and Hedges, Fuzzy Sets and Systems 158 (4) (2007) 436-451.

8. Nguyen Cat Ho and Nguyen Van Long - Fuzziness Measure on Complete Hedge Algebras and Quantifying Semantics of Terms in Linear Hedge Algebras, Fuzzy Sets and Systems 158 (4) (2007) 452-471.

9. Cat Ho Nguyen, Van Nam Huynh, Pedrycz W. - A Construction of Sound Semantic Linguistic Scales Using 4-Tuple Representation of Term Semantics, Int. J. Approx. Reason 55 (2014) 763-786.

10. Cat Ho Nguyen, Thai Son Tran, Dinh Phong Pham - Modeling of a semantics core of linguistic terms based on an extension of hedge algebra semantics and its application, Knowledge Based Systems 67 ( 2014 ) 244-262.

11. Zadeh L. - Fuzzy logic $=$ computing with words, IEEE Transactions on Fuzzy Systems 94 (2) (1996) 103-111.

12. Herrera F and Martínez L. - A 2-Tuple Fuzzy Linguistic Representation Model for Computing with Words, IEEE Transactions on Fuzzy Systems 8 (6) (2000) 746-752. 
13. Martinez L, Ruan D, Herrera F. - Computing with words in decision support systems: an overview on models and applications, International Journal of Computational Intelligence Systems 3 (4) (2010) 382-395.

14. Martínez L, Herrera F. - An overview on the 2-tuple linguistic model for Computing with Words in Decision Making: Extensions, applications and challenges, Information Sciences 207 (1) (2012) 1-18,

15. Hai Le. Bui, Duc Trung Tran, Nhu Lan Vu - Optimal fuzzy control using hedge algebras of a damped elastic jointed inverted pendulum, Vietnam Journal of Mechanics 32 (4) (2010) 247-262.

16. Hai Le Bui, Dong Anh Nguyen, Duc Trung Tran, Nhu Lan Vu - Application of hedge algebra-based fuzzy controller to active control of a structure against earthquake, Struct. Control and Health monit. 20 (2013) 483-495.

17. Hai Le Bui, Duc Trung Tran, Nhu Lan Vu - Optimal fuzzy control of inverted pendulum, J. Vib. and Control 18 (14) (2012a) 2097-2110.

18. Hai Le Bui, Dinh Duc Nguyen, Nhu Lan Vu and Duc Trung Tran - A study on the application of hedge algebras to active fuzzy control of a seism-excited structure, J. Vib. and Control 18 (14) (2012b) 2186-2200.

19. Xuan Viet Le, Cat Ho Nguyen, Nhu Lan Vu - Optimal hedge-algebras-based controller: Design and Application, Fuzzy Set and Syst. 159 (2008) 968-989.

20. Nguyen Cat Ho, Vu Nhu Lan, Le Xuan Viet - Quantifying Hedge Algebras, Interpolative Reasoning Method and its Application to Some Problems of Fuzzy Control, WSEAS TRANSACTIONS on COMPUTERS 5 (11) (2006) 2519-2529.

21. Guclu R, Yazici H - Vibration control of a structure with ATMD against earthquake using fuzzy logic controllers, Journal of Sound and Vibration 318 (2008) 36-49.

22. Hai Le Bui, Cat Ho Nguyen, Pedrycz Witold, Duc Trung Tran and Nhu Lan Vu - Active control of earthquake-excited structures with the use of hedge-algebras-based controllers, Tạp chí khoa học công nghệ (Journal of Science and Technology) 50 (6) (2012) 705-734.

\title{
TÓM TẮT
}

\section{ĐẠI SỐ GIA TƯ’, NGŨ๋ NGHĨA CỦA THÔNG TIN NGÔN NGŨ̉ MỜ VÀ TRIẾN VỌNG ÚNG DỤNG}

\author{
Cat Ho Nguyen ${ }^{1, *}$, Thai Son Tran ${ }^{1}$, Nhu Lan $\mathrm{Vu}^{1,2}$
}

${ }^{1}$ Viện Công nghệ Thông tin, Viện HLKHCNVN, 18 Hoàng Quốc Việt, Cầu Giấy, Hà Nội

${ }^{2}$ Đại học Thăng Long, Nghiêm Xuân Yêm, Hoàng Mai, Hà Nội, Việt Nam

*Email: ncatho@gmail.com

Mục tiêu của bài báo tổng quan là muốn chứng tỏ đại số gia tử thực sự mô hình hóa được ngữ nghĩa đứng đắn của từ ngôn ngữ của các biến, dựa trên cơ sở lập luận rằng ngữ nghĩa định tính vốn có của chúng phải biểu thị qua các quan hệ thứ tự giữa các từ của cùng một biến ngôn ngữ. Ngữ nghĩa như vậy được hình thành trong thực tiễn do nhu cầu trong quá trình lấy quyết 
định trong cuộc sống hàng ngày của con người. Đặc điểm mô hình hóa ngữ nghĩa của từ ngôn ngữ bằng quan hệ thức tự làm cho cách tiếp cận đại số khác biệt hoàn toàn các cách tiếp cận hiện tại và làm cho đại số gia tử trở thành lí thuyết đầu tiên có thể thao tác trực tiếp trên các từ ngôn ngữ. Chúng tôi làm sáng tỏ từng bước những đặc trưng và các tính chất khác biệt biểu thị qua các quan hệ thứ tự trong cách tiếp cận này và qua đó chứng tỏ rằng cách tiếp cận là đúng đắn và là cơ sở bảo đảm tính hiệu quả trong việc bước đầu giải quyết các bài toán ứng đụng. Qua đó chứng tỏ đại số gia tử có nhiều hứa hẹn trong việc phát triển các phương pháp luận để giải quyết các bài toán thuộc các lĩnh vực ứng dụng khác nhau. Để làm sáng tỏ các khẳng định như vậy, chúng tôi sẽ tổng kết các kết quả ứng dụng của đại số gia tử trong một số vấn đề thuộc lĩnh vực khai phá tri thức và điều khiển mờ.

Tù khóa: ngữ nghĩa dựa trên thứ tự, tính mờ của từ ngôn ngữ; ngữ nghĩa dựa trên tập mờ, hệ mờ dựa trên trí thức luật, bài toán phân lớp, điều khiển mờ. 